AperTO - Archivio Istituzionale Open Access dell'Università di Torino

Space experiment TUS on board the Lomonosov satellite as pathfinder of JEM-EUSO

This is a pre print version of the following article:

Original Citation:

Availability:

This version is available http://hdl.handle.net/2318/1578499

since 2016-06-30T15:30:19Z

Published version:

DOI:10.1007/s10686-015-9465-y

Terms of use:

Open Access

Anyone can freely access the full text of works made available as "Open Access". Works made available under a Creative Commons license can be used according to the terms and conditions of said license. Use of all other works requires consent of the right holder (author or publisher) if not exempted from copyright protection by the applicable law. 


\title{
Space Experiment TUS on Board the Lomonosov Satellite as Pathfinder of JEM-EUSO
}

\author{
The JEM-EUSO Collaboration
}

Received: date / Accepted: date

\begin{abstract}
Space-based detectors for the study of extreme energy cosmic rays (EECR) are being prepared as a promising new method for detecting highest energy cosmic rays. A pioneering space device - the "tracking ultraviolet setup" (TUS) - is in the last stage of its construction and testing. The TUS detector will collect preliminary data on EECR in the conditions of a space environment, which will be extremely useful for planning the major JEMEUSO detector operation.
\end{abstract}

Keywords air-shower fluorescence telescope · JEM-EUSO · Pathfinder

\section{Introduction}

Existing ground-based experimental arrays do not collect statistically significant data on EECR - especially beyond the GZK energy limit. New methods of EECR observation, with two orders of magnitudes larger exposure, are needed for the solution of this problem. The method of EAS fluorescence observation

Contribution to the special issue 'JEM-EUSO' of Experimental Astronomy

JEM-EUSO Collaboration

The full author list and affiliations are given at the end of the paper; corresponding authors:

M.I. Panasyuk E-mail: panasyuk@sinp.msu.ru

D.V. Skobeltsyn Institute of Nuclear Physics of M.V. Lomonosov Moscow State University, Russia

B.A. Khrenov E-mail: bkhrenov@yandex.ru

D.V. Skobeltsyn Institute of Nuclear Physics of M.V. Lomonosov Moscow State University, Russia

T. Ebisuzaki E-mail: ebisu@postman.riken.go.jp

RIKEN Advanced Science Institute, Japan

P.A. Klimov E-mail: pavel.klimov@gmail.com

D.V. Skobeltsyn Institute of Nuclear Physics of M.V. Lomonosov Moscow State University, Russia 
from satellites, as proposed by Linsley \& Benson [1], promises to become such a technique. Alternative optical designs of space detectors were suggested from the beginning: First, wide field-of-view lens optics (first the OWL project [2], then the EUSO project [3] and now the JEM-EUSO project [4]), and second, mirror optics (KLYPVE project) based on the experience of producing large area concentrators for solar generators (Russian initiative of SINP MSU and RSC Energia [5,6] which now is realized as Tracking Ultraviolet Set-up (TUS) prototype of KLYPVE detector by SINP MSU, JINR-Dubna with participation of Universities in Korea and Mexico $[7,8])$. The TUS detector will be launched on board the Lomonosov satellite as part of the instrumentation aimed for studies of Extreme Phenomena in the Universe.

\section{Advantages and disadvantages of space-based EECR detectors}

Space detectors have the following advantages:

1. EECR particle tracks can be observed in a huge area of the Atmosphere, owed to the great distance from the detector to the atmosphere. For example, at the orbit height of the ISS (350-400 km), the JEM-EUSO detector will survey $2 \cdot 10^{5} \mathrm{~km}^{2}$ area of the Atmosphere.

2. In one (or more) year of its in-orbit operation, the detector observes the entire celestial sphere. This will allow the distribution of EECR sources to be studied, despite possible inaccuracy in the determination of the primary particle energy. An unavoidable difference in the absolute value of the energy measured by different ground-based arrays causes a difference in EECR intensity in different sky regions covered by different arrays.

At the same time, detectors on board the satellites encounter a variety of difficulties:

1. Observation of EAS from a distance approximately 10 times greater than ground based experiments requires higher sensitivity and resolution of the optical system, including the photon detector. Desirable resolution of one detector pixel should be equal to the diameter of the lateral electron distribution in a shower. For a satellite orbit height of $500 \mathrm{~km}$, the angular resolution of the orbital detector should be $0.4-2 \mathrm{mrad}$, which is at least an order of magnitude higher than that of the existing ground-based detectors (20 mrad).

2. Nighttime atmospheric noise in the UV wavelength band $(300-400 \mathrm{~nm})$ used for EAS detection varies on the path of the satellite. The data from the Universitetsky-Tatiana satellites $[9,10]$ gave a scale of such variations: from $3 \cdot 10^{7}-2 \cdot 10^{8}$ photons $\mathrm{cm}^{-2} \mathrm{sr}^{-1} \mathrm{~s}^{-1}$ on moonless nights (lower values are above oceans, higher values are above aurora zones and cities) to $2 \cdot 10^{9}$ photons $\mathrm{cm}^{-2} \mathrm{sr}^{-1} \mathrm{~s}^{-1}$ on nights of full moon. Ground-based arrays operate on moonless nights at specially chosen locations with a noise level lower than $5 \cdot 10^{7}$ photons $\mathrm{cm}^{-2} \mathrm{sr}^{-1} \mathrm{~s}^{-1}$. 


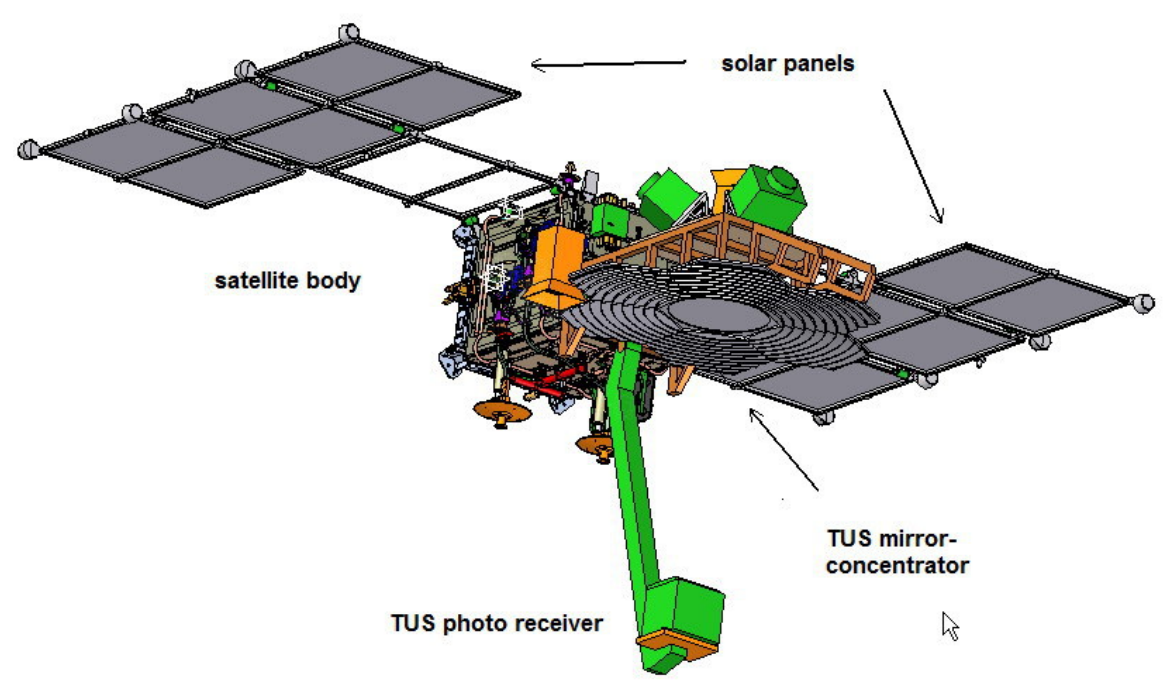

Fig. 1 Detector TUS on board of the Lomonosov satellite.

3. Impulsive noise from lightning and accompanying high altitude discharges will add to the average noise level.

4. Technology of an orbital fluorescence detector should satisfy complex conditions of space operation. Bearing these difficulties in mind, a program for the gradual conversion of a UV fluorescence detector that operated at ground level to a space version was started [6]. The TUS detector is the first, comparatively "simple" instrument that will verify the reliability and stability of an optical system and photo detector design for an operation in space.

\section{The Space Detector TUS}

The detector consists of two main parts: a mirror concentrator, with an area of $\sim 2 \mathrm{~m}^{2}$, and a photo detector composed of 256 pixels, located at the mirror focus (Fig. 1 and 2). The TUS technological parameters are: mass $\approx 60 \mathrm{~kg}$, power consumption $\approx 65 \mathrm{~W}$, and data rate 250 Mbytes/day. The mirror-concentrator is designed as a combination of a central parabolic mirror and 11 parabolic rings focusing a parallel beam on one focal point. In this design, the thickness of the mirror construction is small $(3 \mathrm{~cm})$, which is important for the mirror implementation into the satellite construction. The mirror focal distance is 1.5 $\mathrm{m}$. The mirror is cut into hexagonal segments with a diameter of $66 \mathrm{~cm}$. Mirror segments are made of carbon plastic strengthened by honey comb aluminum plate so that the mirror construction is temperature stable in a wide range of temperatures. The mirror surface is obtained as plastic replicas of aluminum press forms (one for the central mirror part and one for the 6 lateral parts). 


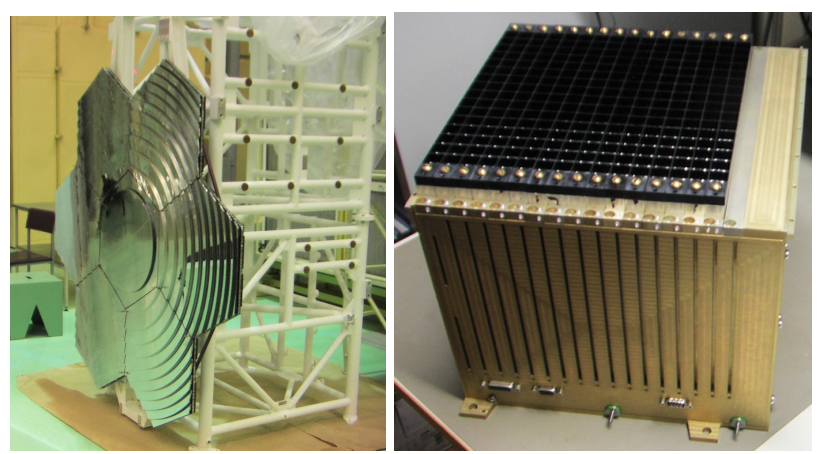

Fig. 2 The TUS mirror concentrator and photo detector.

The plastic mirror surface is covered by aluminum film and protected by a $\mathrm{MgF}_{2}$ coat in a vacuum evaporation process. Reflectivity of the mirror surface at wavelength $350 \mathrm{~nm}$ (average for the atmosphere fluorescence) is $85 \%$. TUS mirror passed various space qualification and optical tests. Those tests show the stability of the optical quality of the mirror in space conditions. The expected lifetime of the mirror is more than 3 years. Photo detector pixels are photomultiplier tubes PMT R1463 of Hamamatsu with multi-alcali cathode of $13 \mathrm{~mm}$ diameter. The quantum efficiency of the PMT cathode is $20 \%$ at $350 \mathrm{~nm}$. A PMT's multi-alcali cathode (instead of bi-alcali one usually used in ground-based fluorescence detectors) was chosen for operation in a wider range of temperatures, in which the cathode operates in the linear regime. To make the detector field of view (FOV) uniformly filled with pixels, light guides, with square entrance $(15 \times 15 \mathrm{~mm})$, and circle output, adjusted to PMT cathode, were used. TUS electronics [11,12] measure the integral number of PMT photoelectrons digitized by ADC every interval of $0.8 \mu \mathrm{s}$ in every pixel. PMTs gain follows intensity of the UV received by pixel in a time interval of $100 \mathrm{~ms}$ so that digitized photoelectrons number is coded by 2 numbers: code $N$ of ADC and code $M$ of PMT gain (high voltage). In this mode, pixel PMT operates night and day keeping sensitivity for years. Two years of operation with $10 \%$ stability of gain was proved in measurements of Tatiana satellite pixels. It should be mentioned that TUS electronics is different to JEM-EUSO electronics, currently under testing in other JEM-EUSO prototypes. The qualification testing of phototubes was done with the hardware and software experimental setup (test bench), used successfully by JINR group for PMTs test of ATLAS Tile Calorimeter at LHC. After testing, PMTs with similar gain were grouped in 16 tube clusters. Data from each tube in the cluster are digitized by one ADC and then analyzed and memorized by the cluster FPGA. Final detector triggering and memorizing of all data is done by TUS central FPGA. The information volume of one EAS data is $\approx 100$ Kbytes. The expected volume of the EAS data from one day transmitted to the mission center is 250 Mbytes. 


\section{Detector performance}

The performance of the TUS detector was simulated taking into account parameters of the real TUS mirror-concentrator and the TUS electronics by use of the ESAF program designed for the JEM-EUSO space detector. Focusing of the mirror-concentrator was checked during the experimental measurements of mirror PSF (point spread function). In Fig. 3, results of PSF measurements (right panel) are compared with the PSF of an "ideal" mirror (left panel). Light beam was tried at 8 different azimuthal $(\phi)$ and 4 polar $(\theta)$ angles $\left(\theta=0^{\circ}, 1.5^{\circ}, 3^{\circ}, 4.5^{\circ}\right)$. As follows from Fig. 3 , the real mirror PSF is not a point even at small polar angles. Nevertheless, the diameter of PSF is less than the pixel size in the $9^{\circ} \mathrm{FOV}$.
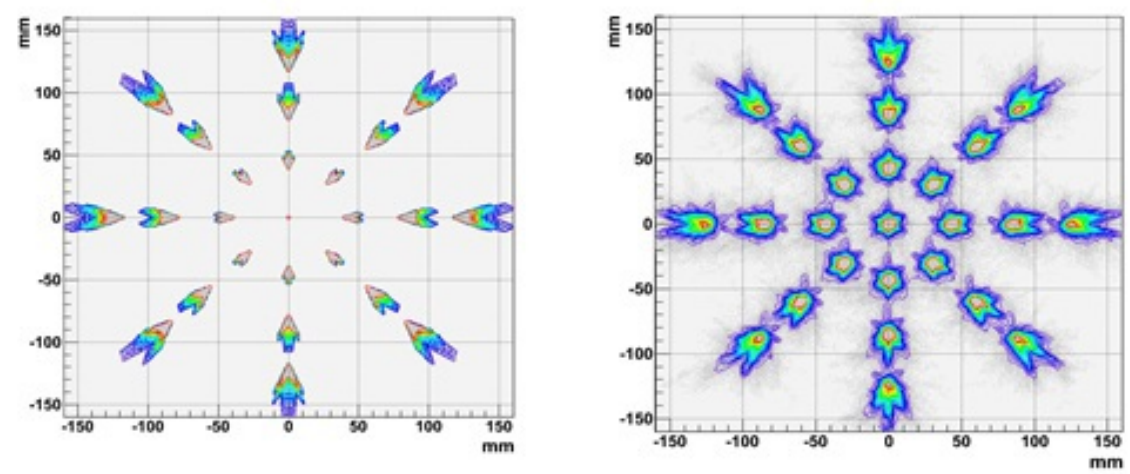

Fig. 3 Focal spots of "ideal" mirror. Focal spots of "real" mirror.

Real mirror EAS signals (photon numbers in pixels detecting EAS cascade curve) were simulated and compared with signals for an "ideal" mirror. Typical results of such simulations are demonstrated in Fig. 4, where the percentage of photons received by the mirror and distributed in pixels is presented for EAS zenith angle $75^{\circ}$. Photon distribution along the EAS cascade curve for "ideal" and real mirrors shows little difference, which shows a good quality of the mirror.

The accuracy of EAS measurements by the TUS detector depends on the level of the night atmosphere noise and on primary particle energy. For primary energy $10^{20} \mathrm{eV}$, zenith angle $75^{\circ}$ and intensity of the atmosphere glow $5 \cdot 10^{7}$ photons $/ \mathrm{cm}^{2} \mathrm{~s}$ sr the pixel signals are presented in Fig. 5 as ratios of signal-tonoise RMS. It shows that the EAS track will be measured by the TUS detector at a signal level of $>3$ RMS in 5-6 pixels. This result confirms the EECR trigger conditions proposed in the earlier work [11]. The TUS EECR trigger operates in 2 stages: in the first stage, pixels with a signal $A$-times larger than noise RMS are selected; in the second stage, events with $B$ neighbour pixels having signal $\geq A$ and line up in space and time, are selected. The final trigger 

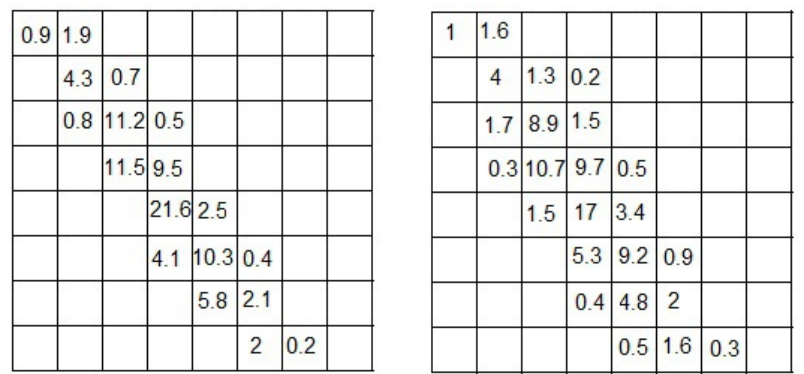

Fig. 4 Percentage of EAS photon numbers focused by mirror to one pixel. Left panel: "ideal" mirror, right panel: real mirror. Only pixels with percentage more than $0.2 \%$ are shown.
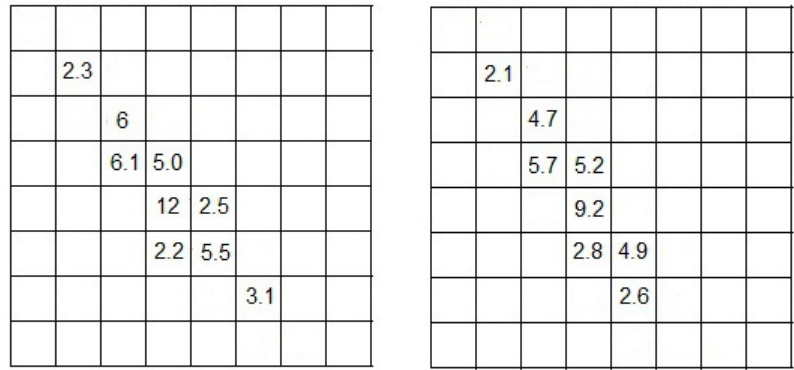

Fig. 5 EAS pixel signals in number of RMS values of noise. Left panel: "ideal" mirror, right panel for real mirror.

begins recording data from all pixels. Numbers $A$ and $B$ will be controlled from the mission center in a compromise between trigger rate, limited by the upper volume of information to be transmitted per day, and the TUS detector energy threshold.

\section{Present data on background effects in measuring EECR from space}

One of the TUS mission tasks is the measurement of the atmosphere UV background radiation capable of imitating the useful events of EECR. In preparation of TUS operation, the experimental data on atmospheric radiation obtained in orbital measurements by Universitetsky-Tatiana (Tatiana-1) and Universitetsky-Tatiana-2 (Tatiana-1) satellites are used. It is worthwhile to present Tatiana- 1 and Tatiana- 2 data in connection with the TUS prototype, where the background effects will prevail. Experimental results of "Tatianas" 
on UV average intensity are presented in Fig. 6 . The data in this figure are presented for the moonless nights of winter 2009-2010, when borders in latitude of the Earth night side were $30^{\circ} S-60^{\circ} \mathrm{N}$. Intensity $J$ of the atmosphere glow varies in a wide range from $3 \cdot 10^{7} \mathrm{ph} / \mathrm{cm}^{2} \mathrm{~s}$ sr to $2 \cdot 10^{8} \mathrm{ph} / \mathrm{cm}^{2} \mathrm{~s}$ sr. It is well known that atmosphere glow is radiated in a comparatively narrow layer of upper atmosphere (lower ionosphere) at heights of 80-100 km. When the fluorescence detector looks down from satellite orbit to nadir, it directly detects the atmosphere glow, practically without absorption in the upper atmosphere. It should be mentioned that in ground-based EAS detectors looking horizontally, the atmosphere glow from heights $80-100 \mathrm{~km}$ is strongly absorbed and ground-based detectors, operating in special low noise regions and on moonless nights, operates at lower background $\left(J<5 \cdot 10^{7} \mathrm{ph} / \mathrm{cm}^{2} \mathrm{~s} \mathrm{sr}\right)$. In the geometry of orbital detectors, the upper atmosphere glow in some places increases the background noise up to $\approx 10^{8} \mathrm{ph} / \mathrm{cm}^{2} \mathrm{~s}$ sr.

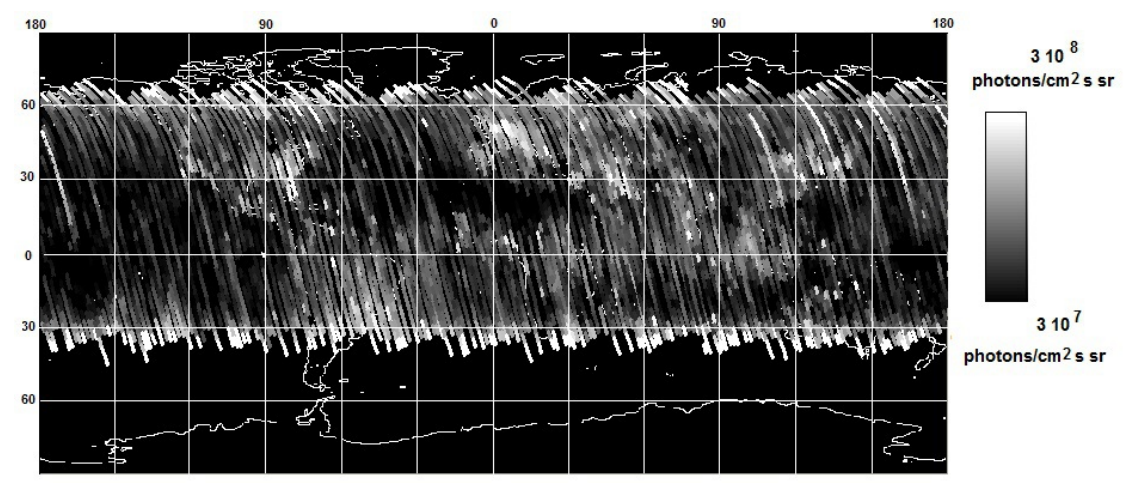

Fig. 6 Global map of night atmosphere glow intensity in UV wavelength band (240-400 $\mathrm{nm})$ as measured by detector of "Universitetsky-Tatiana-2" satellite [10].

Taking into account these data on the atmosphere glow, the exposure of TUS detector with FOV of $9^{\circ}$ was estimated [12]. Efficiency of EECR event selection is close to $100 \%$ for $E>300 \mathrm{EeV}$ and those events will be collected with exposure of $12000 \mathrm{~km}^{2} \mathrm{sr}$ yr in 3 years of in-orbit operation. Events with lower energies will be detected with less efficiency (exposure). For steep energy spectrum of EECR after the GZK limit (integral spectrum exponent $\approx 4$ for energies $E>50 \mathrm{EeV}$ ), the statistics with minimum threshold energy $(\sim 70 \mathrm{EeV})$ will be determined by real exposure at the darkest regions of the Earth: above the Pacific Ocean, deserts and part of Siberia (Fig. 6). With such limited exposure, the TUS detector will not make a breakthrough in the problem of EECR origin. Its aim is to check the EAS fluorescence detector performance in a space environment. In orbital flight, the TUS detector will operate above some more intense sources of background glow: aurora lights, city lights, some unknown sporadic lights. Experimental results of Tatiana-1 


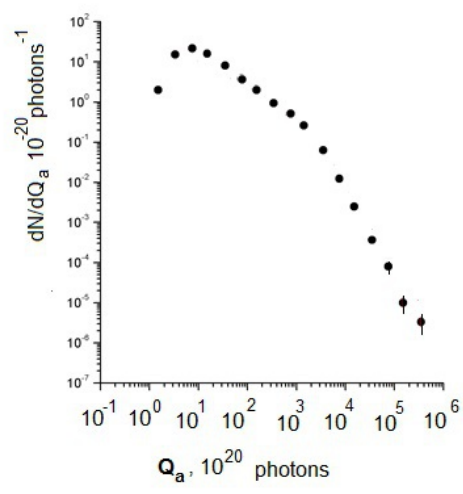

Fig. 7 Photon number distribution of UV flashes [13]

satellites showed that these higher intensity glow sources are not large in size of atmosphere area and do not greatly affect the exposure of the detector. Other sources of background in orbital EECR measurements are short UV flashes (duration of 1-100 ms), the origin of which is related to electrical discharges in the atmosphere. The last achievement in studying this background is data from Tatiana-2 satellite. The UV-detector of this satellite operated in conditions close to the orbital EECR detector, measuring the temporal structure of flashes in the atmosphere area of thousands of square $\mathrm{km}$ in nadir direction. Measurements were done for a wide range of photon number $Q_{a}$ in the atmosphere UV flash: from $Q_{a}=10^{21}$ up to $Q_{a} \approx 10^{25}$ where tens of events were registered. The main features of flashes with $Q_{a}>10^{23}$ - their duration of 10-100 ms, their global distribution concentrated in equatorial region above continents - suggested that those flashes are either lightning itself or transient luminous events (TLE) generically related to lightning. Those bright flashes will be easily separated from EAS fluorescent signals due to their long duration and enormous number of photons (to compare with EAS parameters: duration of not more than $0.1 \mathrm{~ms}$ and number of UV photons $Q_{a} \approx 10^{16}$ for $E=100 \mathrm{EeV})$.

The background of dim and short flashes $\left(Q_{a} \approx 10^{21}-10^{23}\right.$, duration $\approx 1 \mathrm{~ms}$ ) observed by Tatiana-2 detector is more dangerous for imitation of EECR events. In Fig. 7, the flash event distribution over photon numbers $Q_{a}$ is presented. One can see that dim events, with a small photon number $\left(Q_{a}<10^{22}\right)$, are an important part of all events. Global distribution of dim and bright flashes measured in [13] were found different, Fig. 8.

Bright flashes are concentrated in the equatorial region above continents in agreement with lightning activity. Their rate in these regions is of the order of $10^{-3} \mathrm{~km}^{-2} \mathrm{hr}^{-1}$. Dim flashes are distributed more uniformly, with the rate of $\approx 10^{-4} \mathrm{~km}^{-2} \mathrm{hr}^{-1}$. Rates of both kinds of flashes are much higher than the expected rate of EECR events: $\approx 10^{-6} \mathrm{~km}^{-2} \mathrm{hr}^{-1}$. This, a problem of distinguishing EAS flashes from atmospheric flashes could be complicated. 

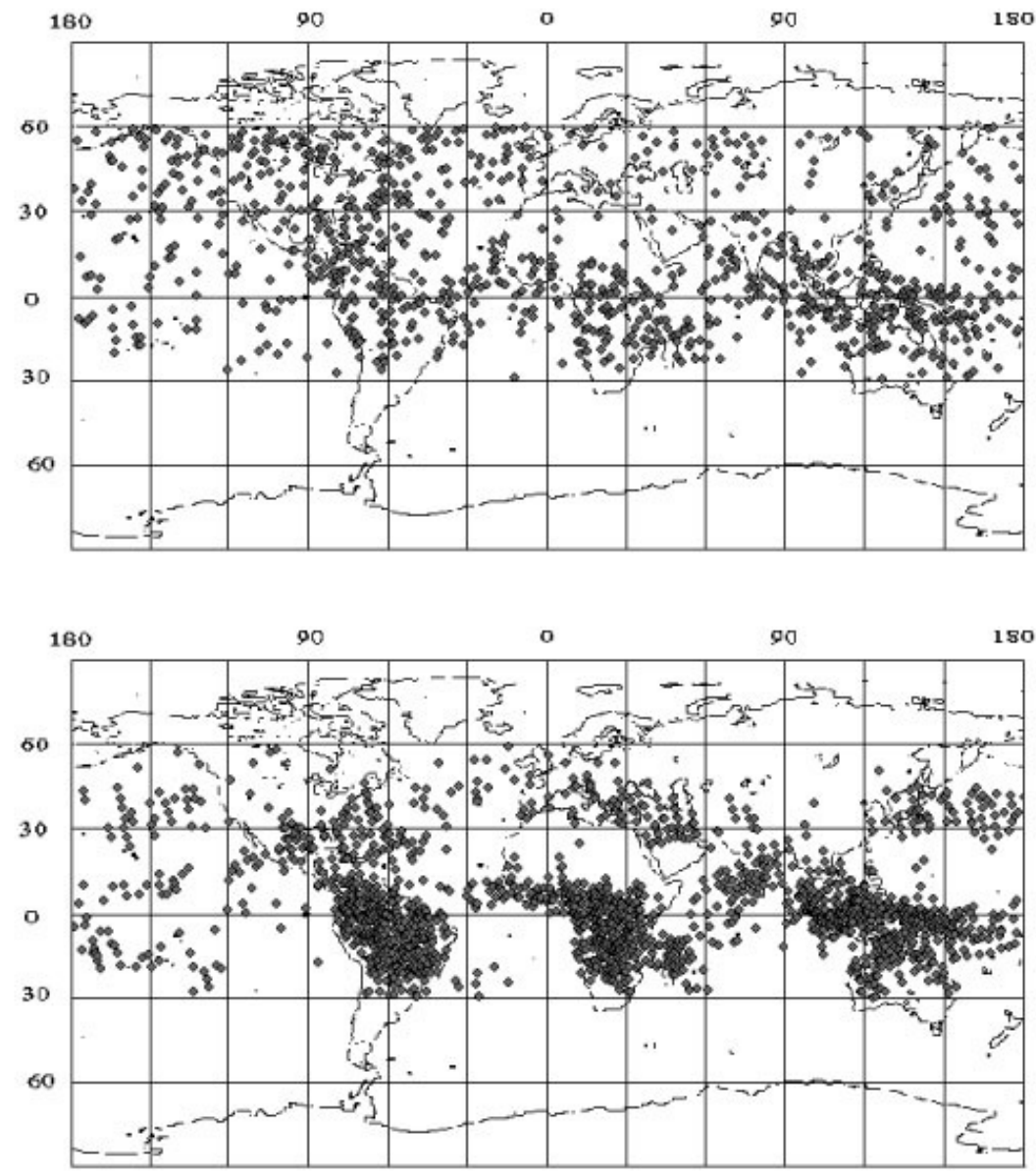

Fig. 8 Global distribution of dim (upper panel, $Q_{a}<5 \cdot 10^{21}$ ) and bright (bottom panel, $Q_{a}>10^{23}$ ) UV flashes [13].

There is a great difference in the photon number of EECR flashes $\left(Q_{a} \approx\right.$ $10^{16}$ for $E=100 \mathrm{EeV}$ ) and in the number of photons in atmospheric flashes, even for dim flashes $\left(Q_{a} \approx 10^{16}-10^{22}\right)$. The duration of EECR flashes is much shorter than that of atmospheric flashes $(0.1 \mathrm{~ms}$ for EECR flash and $1 \mathrm{~ms}$ for atmospheric dim flashes) but still the imitation of EECR events by early stage atmospheric flashes is possible. The TUS operation will give important data on the background effect of atmospheric flashes. 


\section{Conclusion}

Operation of the TUS orbital detector will be an important stage of EECR study by a new method of fluorescent tracking of EAS from space.

Acknowledgements This work is supported partially by the grant of Russian Foundation for Basic Research No. 13-02-12175 ofi-m.

\section{References}

1. Linsley J., and R. Benson (1981). Proc. of the 17th ICRC (Paris, 1981) 8, 145.

2. Streitmatter R.E. et al, Proceedings of Workshop on Observing of Giant Cosmic Ray Air Showers from $>10^{20} \mathrm{eV}$ Particles from Space. AIP Conf. Proceedings, 433, 95 (1998)

3. Scarsi L. et al, Extreme Universe Space Observatory (EUSO) Proposal for the ESA F2/F3 Missions, (2000).

4. Takahashi, Y. (for JEM-EUSO Collab.) (2008). J. Phys.: Conf. Ser. 120, 062013

5. Garipov G.K. et al . (1998) AIP Conference Proceedings, v. 433, 403-417, 1998.

6. Alexandrov V.V. et al. MSU Bulletin (2000). Nr.6, 33.

7. Khrenov B. A., Panasyuk, M. I., et al. (2001). AIP Conf. Proc. 566, 5.

8. Abrashkin V. et al (2007) Nucl. Phys. B (Proc. Suppl.) 166, 68-71.

9. Sadovnichii V. A., M.I. Panasyuk, et al. (2011). Solar System Research, 45, 1, 3-29.

10. Vedenkin N.N. et al, (2011). JETP, 45, 1.

11. Garipov G.K. et al (2001) AIP Conf Proc . 566, 76

12. Klimov P.A. PhD Thesis, (2009) SINP MSU

13. Garipov G., et al. (2013) J. Geophys. Res., 118, issue 2, 340. doi:10.1029/2012JD017501.

The JEM-EUSO Collaboration

J.H. Adams Jr. ${ }^{m d}$, S. Ahmad ${ }^{b b}$, J.-N. Albert ${ }^{b a}$, D. Allard ${ }^{b c}$, L. Anchordoqui ${ }^{m f}$, V. Andreev ${ }^{m e}$, A. Anzalone ${ }^{d h, d n}$, Y. Arai ${ }^{e v}, \mathrm{~K}$. Asano ${ }^{e t}$, M. Ave Pernas ${ }^{k c}$, P. Baragatti ${ }^{d o}$, P. Barrillon ${ }^{b a}$,

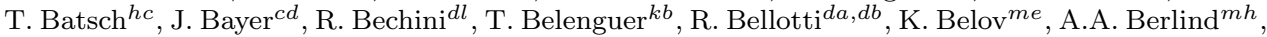
M. Bertaina ${ }^{d k, d l}$, P.L. Biermann ${ }^{c b}$, S. Biktemerova ${ }^{i a}$, C. Blaksley ${ }^{b c}$, N. Blanc ${ }^{l a}$, J. Błęcki ${ }^{h d}$, S. Blin-Bondil ${ }^{b b}$, J. Blümer $^{c b}$, P. Bobik ${ }^{j a}$, M. Bogomilov $^{a a}$, M. Bonamente ${ }^{m d}$, M.S. Briggs ${ }^{m d}$, S. Briz ${ }^{k d}$, A. Bruno ${ }^{d a}$, F. Cafagna ${ }^{d a}$, D. Campana ${ }^{d f}$, J-N. Capdevielle ${ }^{b c}$, R. Caruso ${ }^{d c, d n}$, M. Casolino ${ }^{e w, d i}$, C. Cassardo ${ }^{d k}, d l$, G. Castellini $d d$, C. Catalano ${ }^{b d}$, O. Catalano ${ }^{d h, d n}$, A. Cellino $d k, d m$ M. Chikawa ${ }^{e d}$, M.J. Christl ${ }^{m g}$, D. Cline ${ }^{m e}$, V. Connaughton ${ }^{m d}$, L. Conti $^{d o}$, G. Cordero ${ }^{g a}$, H.J. Crawford ${ }^{m a}$, R. Cremonini ${ }^{d l}$, S. Csorna ${ }^{m h}$, S. Dagoret-Campagne ${ }^{b a}$, A.J. de Castro ${ }^{k d}$, C. De Donato ${ }^{d i}$, C. de la Taille ${ }^{b b}$, C. De Santis ${ }^{d i, d j}$, L. del Peral ${ }^{k c}$, A. Dell'Oro ${ }^{d k, d m}$ N. De Simone $^{d i}$, M. Di Martino ${ }^{d k, d m}$, G. Distratis ${ }^{c d}$, F. Dulucq ${ }^{b b}$, M. Dupieux ${ }^{b d}$, A. Ebersoldt ${ }^{c b}$, T. Ebisuzaki ${ }^{e w}$, R. Engel ${ }^{c b}$, S. Falk ${ }^{c b}$, K. Fang ${ }^{m b}$, F. Fenu ${ }^{c d}$, I. Fernández-Gómez ${ }^{k d}$, S. Ferrarese $^{d k, d l}$, D. Finco ${ }^{d o}$, M. Flamini ${ }^{d o}$, C. Fornaro ${ }^{d o}$, A. Franceschi ${ }^{d e}$, J. Fujimoto $^{e v}$, M. Fukushima ${ }^{e g}$,

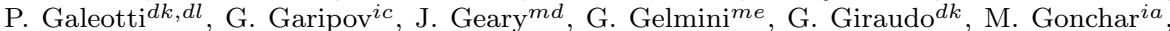
C. González Alvarado ${ }^{k b}$, P. Gorodetzky ${ }^{b c}$, F. Guarino ${ }^{d f, d g}$, A. Guzmán ${ }^{c d}$, Y. Hachisu ${ }^{e w}$, B. Harlov ${ }^{i b}$, A. Haungs ${ }^{c b}$, J. Hernández Carretero ${ }^{k c}$, K. Higashide ${ }^{e r, e w}$, D. Ikeda ${ }^{e g}$, H. Ikeda $^{e p}$,

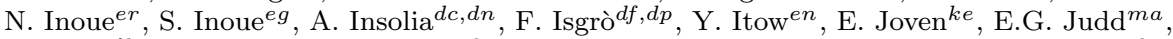
A. Jung $f b$, F. Kajino ${ }^{e i}$, T. Kajino ${ }^{e l}$, I. Kaneko ${ }^{e w}$, Y. Karadzhov ${ }^{a a}$, J. Karczmarczyk ${ }^{h c}$, M. Karus ${ }^{c b}$, K. Katahira ${ }^{e w}$, K. Kawai ${ }^{e w}$, Y. Kawasaki ${ }^{e w}$, B. Keilhauer ${ }^{c b}$, B.A. Khrenov ${ }^{i c}$, Jeong-Sook Kim ${ }^{f a}$, Soon-Wook Kim ${ }^{f a}$, Sug-Whan Kim ${ }^{f d}$, M. Kleifges ${ }^{c b}$, P.A. Klimov ${ }^{i c}$, D. Kolev ${ }^{a a}$, I. Kreykenbohm ${ }^{c a}$, K. Kudela ${ }^{j a}$, Y. Kurihara ${ }^{e v}$, A. Kusenko ${ }^{m e}$, E. Kuznetsov ${ }^{m d}$,

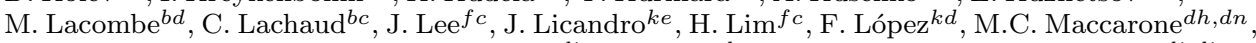

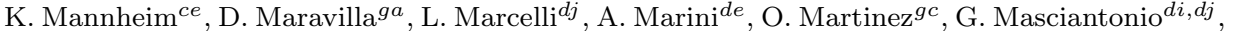
K. Mase ${ }^{e a}$, R. Matev ${ }^{a a}$, G. Medina-Tanco ${ }^{g a}$, T. Mernik $^{c d}$, H. Miyamoto ${ }^{b a}$, Y. Miyazaki ${ }^{e c}$, Y. Mizumoto ${ }^{e l}$, G. Modestino ${ }^{d e}$, A. Monaco ${ }^{d a}, d b$, D. Monnier-Ragaigne ${ }^{b a}$, J.A. Morales de los Ríos ${ }^{k a, k c}$, C. Moretto ${ }^{b a}$, V.S. Morozenko ${ }^{i c}$, B. Mot ${ }^{b d}$, T. Murakamief ${ }^{\text {, M. Nagano }}{ }^{e c}$, M. Nagata ${ }^{e h}$, S. Nagataki ${ }^{e k}$, T. Nakamura ${ }^{e j}$, T. Napolitano de , D. Naumov ${ }^{i a}$, R. Nava ${ }^{g a}$,

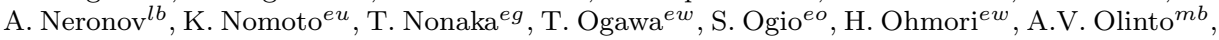




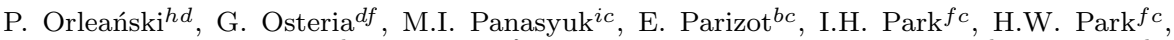
B. Pastircak ${ }^{j a}$, T. Patzak ${ }^{b c}$, T. Paul ${ }^{m f}$, C. Pennypacker ${ }^{m a}$, S. Perez Cano ${ }^{k c}$, T. Peter ${ }^{l c}$, P. Picozza ${ }^{d i}, d j, e w$, T. Pierog ${ }^{c b}$, L.W. Piotrowski ${ }^{e w}$, S. Piraino ${ }^{c d, d h}$, Z. Plebaniak ${ }^{h c}$, A. Pollini $^{l a}$, P. Prat ${ }^{b c}$, G. Prévôt ${ }^{b c}$, H. Prieto ${ }^{k c}$, M. Putis ${ }^{j a}$, P. Reardon ${ }^{m d}$, M. Reyes ${ }^{k e}$, M. Riccide, I. Rodríguez ${ }^{k d}$, M.D. Rodríguez Frías ${ }^{k c}$, F. Ronga ${ }^{d e}$, M. Roth $^{c b}$, H. Rothkaehl $^{h d}$, G. Roudil ${ }^{b d}$ I. Rusinov ${ }^{a a}$, M. Rybczyński ${ }^{h a}$, M.D. Sabau ${ }^{k b}$, G. Sáez Cano ${ }^{k c}$, H. Sagawa ${ }^{e g}$, A. Saito ${ }^{e j}$, N. Sakaki ${ }^{c b}$, M. Sakata ${ }^{e i}$, H. Salazar ${ }^{g c}$, S. Sánchez ${ }^{k d}$, A. Santangelo ${ }^{c d}$, L. Santiago Crúz ${ }^{g a}$, M. Sanz Palomino ${ }^{k b}$, O. Saprykin ${ }^{i b}$, F. Sarazin ${ }^{m c}$, H. Sato ${ }^{e i}$, M. Sato ${ }^{e s}$, T. Schanz ${ }^{c d}$, H. Schieler ${ }^{c b}$, V. Scotti ${ }^{d f, d g}$, A. Segreto ${ }^{d h, d n}$, S. Selmane ${ }^{b c}$, D. Semikoz ${ }^{b c}$, M. Serra ${ }^{k e}$, S. Sharakin ${ }^{i c}$, T. Shibata ${ }^{e q}$, H.M. Shimizu ${ }^{e m}$, K. Shinozakiew, cd, T. Shirahama ${ }^{e r}$, G. SiemieniecOziębło $^{h b}$, H.H. Silva López ${ }^{g a}$, J. Sledd ${ }^{m g}$, K. Słomińska ${ }^{h d}$, A. Sobey ${ }^{m g}$, T. Sugiyama ${ }^{e m}$, D. Supanitsky ${ }^{g a}$, M. Suzuki ${ }^{e p}$, B. Szabelska ${ }^{h c}$, J. Szabelski ${ }^{h c}$, F. Tajima ${ }^{e e}$, N. Tajima ${ }^{e w}$, T. Tajima ${ }^{c c}$, Y. Takahashi ${ }^{e s}$, H. Takami ${ }^{e v}$, M. Takeda ${ }^{e g}$, Y. Takizawa ${ }^{e w}$, C. Tenzer ${ }^{c d}$,

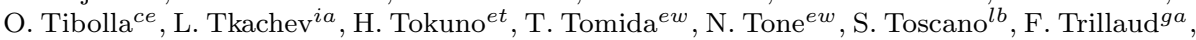

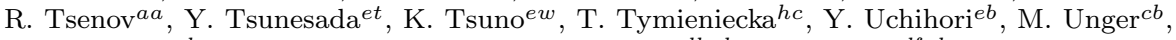
O. Vaduvescuke, J.F. Valdés-Galicia ${ }^{g a}$, P. Vallania ${ }^{d k, d m}$, L. Valore ${ }^{d f, d g}$, G. Vankova ${ }^{a a}$, C. Vigorito ${ }^{d k, d l}$, L. Villaseñor ${ }^{g b}$, P. von Ballmoos ${ }^{b d}$, S. Wada ${ }^{e w}$, J. Watanabe $^{e l}$, S. Watanabe $^{e s}$,

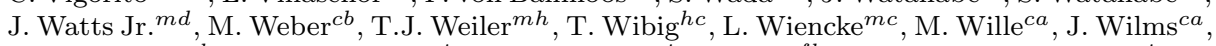
Z. Włodarczyk ${ }^{h a}$, T. Yamamoto ${ }^{e i}$, Y. Yamamoto ${ }^{e i}$, J. Yang ${ }^{f b}$, H. Yano ${ }^{e p}$, I.V. Yashin ${ }^{i c}$,

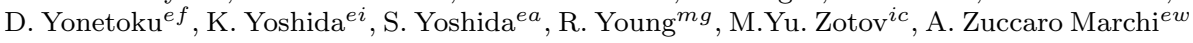
aa St. Kliment Ohridski University of Sofia, Bulgaria

ba LAL, Univ Paris-Sud, CNRS/IN2P3, Orsay, France

${ }^{b b}$ Omega, Ecole Polytechnique, CNRS/IN2P3, Palaiseau, France

bc APC, Univ Paris Diderot, CNRS/IN2P3, CEA/Irfu, Obs. de Paris, Sorbonne Paris Cité, France

${ }^{b d}$ IRAP, Université de Toulouse, CNRS, Toulouse, France

ca ECAP, University of Erlangen-Nuremberg, Germany

${ }^{c b}$ Karlsruhe Institute of Technology (KIT), Germany

${ }^{c c}$ Ludwig Maximilian University, Munich, Germany

${ }^{c d}$ Inst. for Astronomy and Astrophysics, Kepler Center, University of Tübingen, Germany

ce Institut für Theoretische Physik und Astrophysik, University of Würzburg, Germany

da Istituto Nazionale di Fisica Nucleare - Sezione di Bari, Italy

$d b$ Universita' degli Studi di Bari Aldo Moro and INFN - Sezione di Bari, Italy

dc Dipartimento di Fisica e Astronomia - Universita' di Catania, Italy

$d d$ Consiglio Nazionale delle Ricerche (CNR) - Ist. di Fisica Applicata Nello Carrara, Firenze, Italy

de Istituto Nazionale di Fisica Nucleare - Laboratori Nazionali di Frascati, Italy

$d f$ Istituto Nazionale di Fisica Nucleare - Sezione di Napoli, Italy

$d g$ Universita' di Napoli Federico II - Dipartimento di Scienze Fisiche, Italy

$d h$ INAF - Istituto di Astrofisica Spaziale e Fisica Cosmica di Palermo, Italy

di Istituto Nazionale di Fisica Nucleare - Sezione di Roma Tor Vergata, Italy

dj Universita' di Roma Tor Vergata - Dipartimento di Fisica, Roma, Italy

$d k$ Istituto Nazionale di Fisica Nucleare - Sezione di Torino, Italy

$d l$ Dipartimento di Fisica, Universita' di Torino, Italy

$d m$ Osservatorio Astrofisico di Torino, Istituto Nazionale di Astrofisica, Italy

$d n$ Istituto Nazionale di Fisica Nucleare - Sezione di Catania, Italy

do UTIU, Dipartimento di Ingegneria, Rome, Italy

$d p$ DIETI, Universita' degli Studi di Napoli Federico II, Napoli, Italy

ea Chiba University, Chiba, Japan

${ }^{e b}$ National Institute of Radiological Sciences, Chiba, Japan

ec Fukui University of Technology, Fukui, Japan

ed Kinki University, Higashi-Osaka, Japan

ee Hiroshima University, Hiroshima, Japan

ef Kanazawa University, Kanazawa, Japan

eg Institute for Cosmic Ray Research, University of Tokyo, Kashiwa, Japan

eh Kobe University, Kobe, Japan

ei Konan University, Kobe, Japan

ej Kyoto University, Kyoto, Japan

ek Yukawa Institute, Kyoto University, Kyoto, Japan

el National Astronomical Observatory, Mitaka, Japan 
em Nagoya University, Nagoya, Japan

en Solar-Terrestrial Environment Laboratory, Nagoya University, Nagoya, Japan

eo Graduate School of Science, Osaka City University, Japan

$e p$ Institute of Space and Astronautical Science/JAXA, Sagamihara, Japan

eq Aoyama Gakuin University, Sagamihara, Japan

er Saitama University, Saitama, Japan

es Hokkaido University, Sapporo, Japan

et Interactive Research Center of Science, Tokyo Institute of Technology, Tokyo, Japan

eu University of Tokyo, Tokyo, Japan

ev High Energy Accelerator Research Organization (KEK), Tsukuba, Japan

ew RIKEN, Wako, Japan

$f a$ Korea Astronomy and Space Science Institute (KASI), Daejeon, Republic of Korea

$f b$ Ewha Womans University, Seoul, Republic of Korea

$f c$ Sungkyunkwan University, Seoul, Republic of Korea

$f d$ Center for Galaxy Evolution Research, Yonsei University, Seoul, Republic of Korea

ga Universidad Nacional Autónoma de México (UNAM), Mexico

$g b$ Universidad Michoacana de San Nicolas de Hidalgo (UMSNH), Morelia, Mexico

gc Benemérita Universidad Autónoma de Puebla (BUAP), Mexico

ha Jan Kochanowski University, Institute of Physics, Kielce, Poland

$h b$ Jagiellonian University, Astronomical Observatory, Krakow, Poland

$h c$ National Centre for Nuclear Research, Lodz, Poland

$h d$ Space Research Centre of the Polish Academy of Sciences (CBK), Warsaw, Poland

ia Joint Institute for Nuclear Research, Dubna, Russia

$i b$ Central Research Institute of Machine Building, TsNIIMash, Korolev, Russia

ic Skobeltsyn Institute of Nuclear Physics, Lomonosov Moscow State University, Russia

ja Institute of Experimental Physics, Kosice, Slovakia

ka Consejo Superior de Investigaciones Científicas (CSIC), Madrid, Spain

$k b$ Instituto Nacional de Técnica Aeroespacial (INTA), Madrid, Spain

$k c$ Universidad de Alcalá (UAH), Madrid, Spain

$k d$ Universidad Carlos III de Madrid, Spain

ke Instituto de Astrofísica de Canarias (IAC), Tenerife, Spain

la Swiss Center for Electronics and Microtechnology (CSEM), Neuchâtel, Switzerland

$l b$ ISDC Data Centre for Astrophysics, Versoix, Switzerland

${ }_{l c}$ Institute for Atmospheric and Climate Science, ETH Zürich, Switzerland

ma Space Science Laboratory, University of California, Berkeley, USA

$m b$ University of Chicago, USA

$m c$ Colorado School of Mines, Golden, USA

md University of Alabama in Huntsville, Huntsville, USA

me University of California (UCLA), Los Angeles, USA

$m f$ University of Wisconsin-Milwaukee, Milwaukee, USA

$m g$ NASA - Marshall Space Flight Center, USA

$m h$ Vanderbilt University, Nashville, USA 